\title{
Modelo de Predição de Conforto de Usuários do Transporte Coletivo
}

\author{
Vanessa Barbosa Rolim \\ nessabrolim@gmail.com \\ Programa de Pós-Graduação em Computação Aplicada \\ Universidade do Estado de Santa Catarina \\ Joinville, Santa Catarina, Brasil
}

\author{
Fabiano Baldo \\ fabiano.baldo@udesc.br \\ Programa de Pós-Graduação em Computação Aplicada \\ Universidade do Estado de Santa Catarina \\ Joinville, Santa Catarina, Brasil
}

\begin{abstract}
The small and medium-size cities are facing problems related to mobility that could be avoided by adopting the public transportation system, as buses and trains. However, in many Brazilian cities the use of public transportation is neglected because it is considered uncomfortable, expensive and insecure. To attract passengers for such kind of transportation there are several possible approaches, the promotion of comfort perception is one of those. Several studies have already approached this problem, however, few of them addressed the perception of comfort felt by the passengers using telemetry data collected from the vehicle. Among the works that use such data, none of them applied data mining techniques to abstract a general model of comfort perception. Therefore, this work aims to apply mining techniques over telemetry data collected from vehicles to build a comprehensible model to classify the level of comfort of public transportation passengers. To achieve this objective machine learning techniques were used, centered on decision trees. Due to the complexity of abstracting the model there were constructed three models, one for each acceleration axis that were merged using a meta-classifier responsible to point out the passenger general comfort. The results have reached an accuracy of $85,2 \%$, which can be considered a promising result regarding the difficulties of separating the data source in sets that can better identify the bus drivers behaviour.
\end{abstract}

\section{KEYWORDS}

Aprendizado de máquina, Meta-classificação, Conforto, Transporte público, Telemétrico.

\section{INTRODUÇÃO}

No Brasil, 87\% da frota de veículos terrestre é composta por veículos de transporte particular [1]. A abundância de veículos privados é uma das responsáveis pelos congestionamentos diários e pelo declínio na qualidade do ar nas cidades brasileiras [2]. Ainda que algumas localidades tenham encontrado soluções alternativas para esses problemas, é necessário que outras ações sejam tomadas para a solução completa dessa situação. A organização CIVITAS, que auxilia no planejamento logístico das cidades da União Européia, sugere que o aumento do conforto é de suma importância para a atração de passageiros para o transporte coletivo em detrimento ao transporte individual [3].

No entanto, conforto é um conceito complexo, uma vez que está relacionado à percepção de cada pessoa. Existem diferentes aspectos que podem ser analisados de forma sistemática para inferir a percepção de conforto do passageiro. Como exemplo, é possível elencar a sensação de segurança e a forma de condução do veículo
[4]. A sensação de segurança está relacionada à possibilidade de ocorrência de furtos e assaltos e ao estado de conservação do veículo. Já a forma de condução do veículo diz respeito ao comportamento do condutor nas acelerações e frenagens, e na realização de curvas e excesso de velocidade. Este trabalho foca na influência da forma de condução do motorista sobre a percepção de conforto sentida pelo passageiro.

Diversos estudos sugerem maneiras para inferir o conforto com base nas acelerações percebidas pelo usuário [5][6][7][8][9], entretanto, a maioria foca na influência da aceleração horizontal do veículo. Uma análise mais abrangente dos fatores cinéticos que afetam direta ou indiretamente o conforto dos passageiros ainda precisa ser realizada. Portanto, este trabalho apresenta a seguintes pergunta de pesquisa: Como mensurar o grau de conforto dos passageiros de transporte coletivo por meio da avaliação do comportamento de direção do motorista utilizando a trajetória do veículo enriquecida com dados de sensores coletados durante a viagem? Para responder essa pergunta são utilizadas técnicas de mineração de dados, por meio do treinamento de árvores de decisão.

Uma árvore de decisão é uma ferramenta de suporte à decisão que expressa regras e condições para que um determinado estado seja atingido. São utilizadas para diversos fins e, entre eles, para a previsão de um resultado baseado na análise de dados de entrada [10]. Portanto, este trabalho tem por objetivo construir um modelo capaz de classificar a percepção de conforto dos passageiros do transporte coletivo com base em dados cinéticos e de telemetria do veículo por meio da indução supervisionada de árvores de decisão.

Uma das maiores dificuldades na classificação do conforto associado ao comportamento dos motoristas está na coleta de informações que suportem essa análise. Entretanto, essas informações podem ser coletadas por meios de sensores agregados em dispositivos embarcados conectados ao barramento de dados do veículo. Outra dificuldade está associada a classificação de conforto. Como as árvores de decisão são técnicas de aprendizados de máquina supervisionadas, é necessário que os dados de treinamento estejam classificados a priori. Portanto, de posse dos dados de telemetria do veículo é necessário inferir por meio da percepção dos passageiros qual o nível de conforto por eles observado sobre a viagem realizada. As percepções dos passageiros podem ser colhidas por meio de entrevistas e questionários e, posteriormente, serem associadas aos dados cinéticos do veículo. Dessa forma, as trajetórias do veículo enriquecidas com dados de sensores e anotadas com as percepções de conforto dos passageiros podem ser utilizadas para induzir um modelo que caracterize o conforto do passageiro quando considerada a forma de condução do motorista. 
Os dados utilizados neste trabalho foram coletados em veículos de transporte coletivo na cidade de Joinville, Brasil. A pesquisa se baseia na abordagem Pesquisa-Ação, na qual os resultados dos experimentos guiam a evolução iterativa e incremental do modelo que passa por avaliações quantitativas para mensurar sua acurácia a medida em que ele é aprimorado.

As próximas seções são organizadas da seguinte forma. A seção 2 define os termos e notações utilizadas neste estudo. A seção 3 apresenta os trabalhos relacionados. A seção 4 descreve a forma de concepção do modelo proposto. A seção 5 apresenta os resultados dos testes da aplicação do modelo proposto. Por fim, a seção 6 destaca as conclusões e trabalhos futuros.

\section{DEFINIÇÕES}

Esta seção descreve as definições necessárias para o entendimento dos conceito utilizados neste trabalho.

Definição 1 - Trajetória: Uma trajetória $T$ é um conjunto de pontos temporalmente ordenados $T=\left\{p_{1}, p_{2}, \ldots, p_{n}\right\}$, em que $p_{i}$ representa um ponto da trajetória [11]. O início e o fim de uma trajetória são definidos de acordo com o contexto em que a trajetória é analisada.

Definição 2 - Ponto: Um ponto representa a localização de um objeto em um momento, tal que $p=\left(c_{i}, t_{i},\left[A_{i}\right]\right)$, onde $c_{i}$ é a coordenada de um objeto, $t_{i}$ o instante de tempo em que a coleta da coordenada ocorreu, e $\left[A_{i}\right]$ é uma lista de atributos opcionais que enriquecem $p$, como velocidade ou aceleração do objeto [12].

Definição 3 - Coordenada: Uma coordenada $c$ é a identificação do posicionamento espacial de um objeto por meio de um conjunto de dimensões representado por $c=(x, y,[z])$ onde $x$ e $y$ representam o posicionamento bidimensional do objeto no espaço e $z$ é um parâmetro opcional que representa a altura do objeto no espaço [13].

Definição 4 - Velocidade: A velocidade, um atributo opcional da lista $\left[A_{i}\right]$ de um ponto $p$, é uma medida física calculada por meio da divisão da distância $(d)$ percorrida pelo tempo necessário $(t)$ para percorrê-la, ou seja, $v=d / t$ [14].

Definição 5 - Aceleração: A aceleração, um atributo opcional da lista $\left[A_{i}\right]$ de um ponto $p$, é uma medida física que calcula a variação da velocidade $\Delta v=v_{f}-v_{i}$ em função do tempo decorrido $\Delta t=t_{f}-t_{i}$. O cálculo implica na existência de pelo menos dois registros de velocidade, $v_{i}$, velocidade inicial, e $v_{f}$, velocidade final, e o tempo entre um registro e outro, $t_{i}$ e $t_{f}$, respectivamente, ou seja, $a=\Delta v / \Delta t$.

Definição 6 - Aceleração Triaxial: A aceleração triaxial é um conjunto composto por três acelerações, tal que $a_{t}=\left[a_{x}, a_{y}, a_{z}\right]$, onde $x, y$ e $z$ representam aceleração horizontal, lateral e vertical, respectivamente. Essas acelerações estão relacionadas com a direção em que um objeto se desloca e as modificações da velocidade que ocorrem durante seu percurso.

\section{TRABALHOS RELACIONADOS}

Diversos estudos já discutiram a problemática da análise de conforto de passageiros em veículos de transporte coletivo. Esta seção apresenta os trabalhos relacionados mais relevantes encontrados na literatura.
Hassan, Hawas e Ahmed [5] propõe um arcabouço para a medição dos diversos fatores que influenciam na qualidade do transporte coletivo. Esse arcabouço, aplicado em Abu Dhabi, Emirados Árabes, colhe dados quantitativos, como velocidade e aceleração e qualitativos, como percepção de conforto, que são analisados por meio de médias simples e ponderadas, e uma nota de desempenho é atribuída à viagem. Esse método não leva a percepção dos usuários em consideração, portanto, pode apresentar uma visão diferente da sensação de conforto percebida pelo usuário.

Innamaa e Penttinen [6] estuda o efeito da utilização de um sistema de feedback de condução para o motorista, aplicado em Helsinki, Finlândia,. O sistema sugere acelerações e velocidades pré-calculadas de forma a aumentar o conforto dos passageiros. Os autores concluem que os motoristas que tiveram acesso ao sistema receberam avaliações superiores dos passageiros. $\mathrm{O}$ trabalho não apresenta um modelo replicável para a medição de conforto de passageiros, mas demonstra que o feedback instantâneo pode afetar a condução do veículo.

Castellanos e Fruet [7] sugerem um sistema que mede alguns fatores que afetam o conforto de passageiros no transporte coletivo. O sistema é composto por um hardware que coleta e armazena as percepções dos passageiros que são analisadas a posteriori, não existindo feedback em tempo real para o condutor. O sistema foi aplicado em linhas de ônibus na cidade de Campinas, Brasil.

Zhang, Zhou e Zhang [8] propõem um modelo geral de previsão de conforto no transporte coletivo e discute a aplicação em diversas cidades chinesas. O modelo é uma fórmula matemática resultante de diferentes regressões matemáticas baseadas em dados quantitativos e qualitativos. Os autores sugerem que a variável de maior importância para o aumento do desconforto é a aceleração. A fórmula proposta por eles pode não ser válida para outras regiões do mundo que apresentem situações diferentes daquelas encontradas nas cidades testadas.

Eboli, Mazzula e Pungillo [9] sugerem uma abordagem empírica para a análise de conforto. Para tanto, os autores coletaram dados quantitativos de diversas viagens e entrevistaram vários passageiros. No trabalho deles foram analisadas as entrevistas de passageiros que declararam que a viagem foi desconfortável. Após a anotação os dados são utilizados para o cálculo dos limites de conforto. Essa análise é consideravelmente simplificada, pois não analisa como diferentes aspectos de cada viagem podem ter influência entre si.

Park et. al [15] analisam o efeito das vibrações dos veículos enquanto parados sobre o conforto dos passageiros. Para esse experimento, os autores utilizaram um simulador de vibração, de forma que voluntários pudessem ser expostos a vibrações equivalentes às dos veículos reais. Os autores concluem que os limites da ISO 26311:1997 são muito amplos para representar os impactos na avaliação do impacto da vibração no conforto. Ainda, os autores sugerem que os limites devem ser diminuídos para a caracterização de desconforto.

Os estudos tratados nessa seção demonstram que os aspectos cinéticos de uma viagem influenciam diretamente no conforto dos passageiros. Ainda, discutem que as normas atuais para conforto não apresentam limites de conforto que possam ser generalizados de forma a tratar adequadamente o conforto de passageiros de transporte coletivo. 
Sob esse contexto, o estudo em tela usa uma abordagem diferente para classificar dados de telemetria de trajetórias de veículos de transporte coletivo de acordo com o conforto dos passageiros. O método utiliza aprendizado de máquina para classificar trajetórias, com base em percepções de passageiros e grandes massas de dados reais. Dessa forma, é possível diminuir o viés especialista e automatizar a análise de conforto.

\section{MODELO DE CLASSIFICAÇÃO}

A metodologia utilizada na concepção do modelo de classificação do conforto dos passageiros é ilustrada na Figura 1. O processo começa pela etapa de coleta de dados (Figura 1.a), realizada tanto por meio da telemetria quanto pela coleta das percepções dos passageiros. Ele continua com a etapa de pré-processamento (Figura 1.b), que engloba a discretização e formatação dos dados. Ela garante que somente dados válidos sejam considerados no conjunto de dados de treinamento. A próxima etapa (Figura 1.c) realiza a segmentação e anotação das classes de conforto dos dados formatados na etapa anterior. Por fim, na etapa de treinamento (Figura 1.d) o modelo de classificação do conforto é induzido por meio do treinamento das árvores de decisão.

As próximas subseções descrevem em detalhes cada uma das etapas do processo de concepção do modelo.

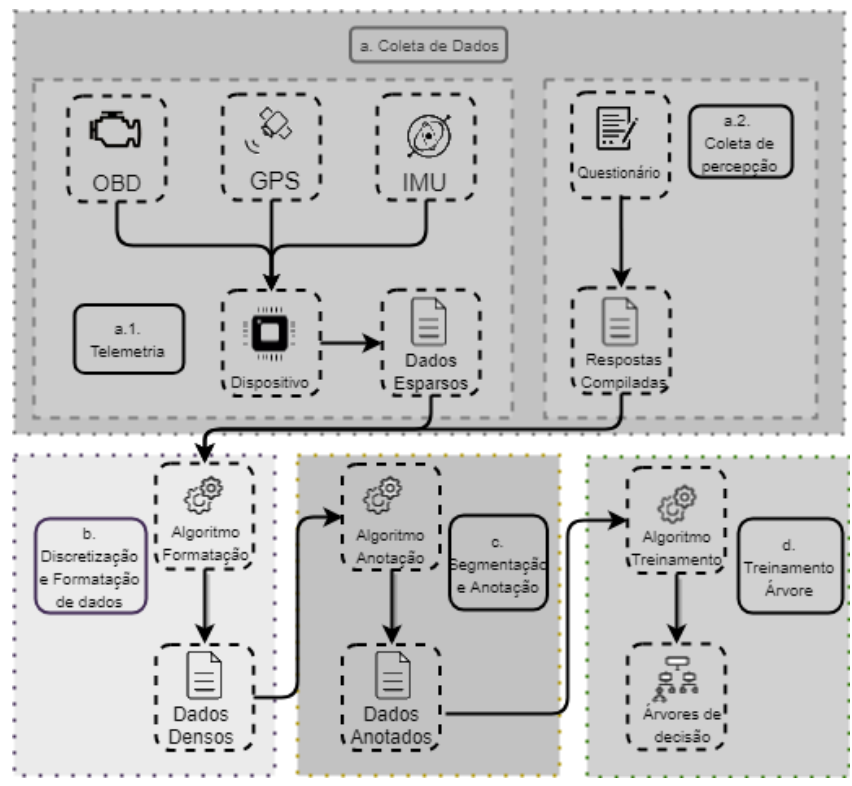

Figura 1: Esquema do método proposto.

\subsection{Coleta de dados}

Os dados de telemetria utilizados neste trabalho foram coletados no âmbito do trabalho de Bayer [16]. Bayer [16] descreve que os dados de transporte coletivo foram coletados por meio de um dispositivo instalado no veículo que recebia e armazenava os dados de GPS (Global Positioning System - Sistema de posicionamento global), OBD (On-board Diagnostic - Diagnóstico a bordo) e IMU (Inertial Measurement Unit - Unidade de medição inercial), sensores responsáveis pela coleta de dados de localização geográfica, informações da unidade de controle do motor e medições inerciais, respectivamente.

Além dos dados descritos acima, é necessário coletar os dados relacionados com a percepção de conforto por parte dos passageiros. Para tanto, um questionário de 7 perguntas foi desenvolvido. O questionário é composto por 3 perguntas descritivas, que objetivaram posicionar geograficamente o trajeto no qual o passageiro viajou e que formaram sua percepção, além de 4 perguntas objetivas na escala de likert, indo de muito confortável a muito desconfortável, que objetivam coletar a percepção do passageiro com relação ao conforto durante a viagem sob a perspectiva de trepidações, curvas, freadas e arrancadas, e da viagem como um todo. Essas percepções estão diretamente relacionadas com os dados dos eixos de aceleração x, y e z.

\subsection{Pré-processamento dos dados de telemetria}

Segundo Bayer [16], os dados coletados são armazenados em um arquivo de texto esparso, em que cada linha contém o momento da coleta e os dados de um sensor. Portanto, é necessário organizálos em um conjunto denso de dados. Além disso, os sensores no dispositivo colhem os dados em diferentes intervalos. Dessa forma, para os dados de maior frequência de coleta, como os dados de acelerômetro, a média e o desvio padrão do período é calculada e esse é o valor considerado para esse sensor.

Além disso, o volume de dados coletados pode ser muito grande, mesmo após as sumarizações realizadas. Para garantir que somente dados que contenham informações relevantes sejam considerados, o conjunto de dados foi tratado para retirar os stay points, pontos de parada do veículo.

\subsection{Anotação dos dados de telemetria com a percepção dos passageiros}

Uma vez que todos os dados coletados foram pré-processados, é necessário associar a eles as suas respectivas classes a fim de dar prosseguimento ao treinamento supervisionado das árvores de decisão. A esse processo dá-se o nome de anotação da percepção. Para tanto, as respostas objetivas dos usuários ao questionário foram estratificadas de 1 a 5 , onde 1 correspondia à opção muito desconfortável e 5 à opção muito confortável. Como as opções estão relacionadas com perguntas sobre o conforto com relação às acelerações triaxiais, os dados de acelerações são classificados em cada viagem de acordo com a nota atribuída pelos passageiros a cada um dos 3 eixos.

Os dados de aceleração utilizados na anotação de percepção estão em módulo, logo as aceleração negativa, ou desaceleração, são consideradas positivas. Dessa forma, a classe Muito Confortável é representada pela parte inferior da distribuição, de zero a $-2 \sigma$ $+\sigma / 2$, em que $\sigma$ representa o desvio padrão do grupo. A classe Razoavelmente Confortável representa os dados que se estendem desde $-2 \sigma+\sigma / 2$ até $-\sigma / 2$, e assim por diante. A Figura 2 apresenta de forma visual as faixas de distribuição das acelerações por classe de conforto.

Como uma mesma viagem do veículo pode ser avaliada por mais de um passageiro, um mesmo ponto da trajetória do veículo pode ter avaliações distintas e, eventualmente, antagônicas. Entretanto, 


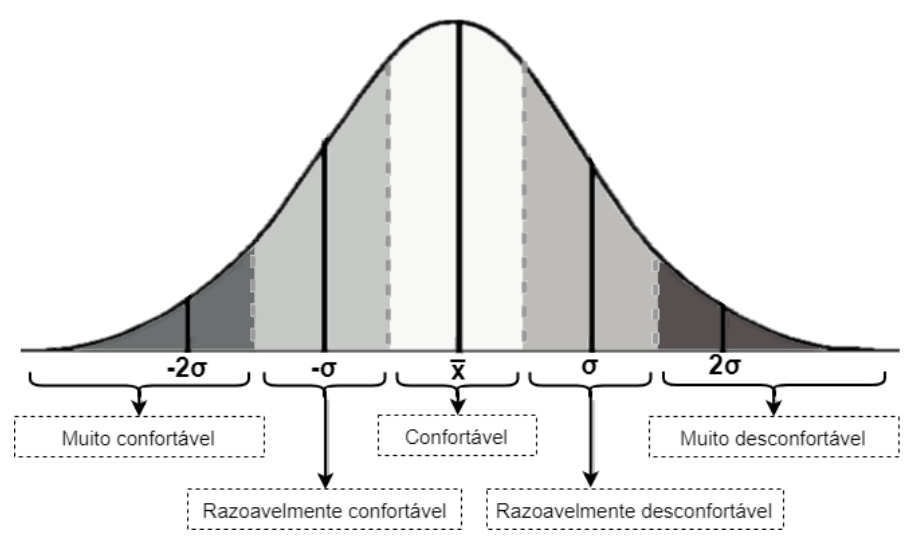

Figura 2: Anotação de percepção de passageiros nas trajetórias com base na distribuição de acelerações em cada eixo, classificadas por eixo.

é importante que cada ponto tenha apenas uma classificação para que o treinamento do modelo não tenha dificuldades em reconhecer o padrão de classificação de um determinado conjunto de valores. Para resolver o problema de pontos com avaliações de conforto diferentes, foi utiliza a média entre as percepções estratificadas com arredondamento para baixo. Por exemplo, se em uma viagem três passageiros diferentes expressaram percepções discordantes, sendo elas confortável, muito confortável e muito desconfortável, onde, de forma numérica, tem-se os valores 1, 3 e 5, calculando-se a média, cujo valor é 4 , o ponto seria classificado como Razoavelmente Confortável.

\subsection{Treinamento das árvores de decisão}

Para realizar o treinamento, os dados anotados com a percepção de conforto foram divididos em dois conjuntos, um usado para a indução das árvores de decisão e o outro para o teste dos modelos criados. Considerando-se que as percepções coletadas estão relacionadas com os eixos de aceleração, x y e z, o resultado do treinamento é um modelo relacionado a cada eixo. Dessa forma, os modelos consideram todas as variáveis disponíveis para o conforto em cada eixo, mencionadas na Seção 4.1, de tal maneira que seja possível considerar outras variáveis além das acelerações.

Como os modelos resultantes do treinamento são três árvores de decisão distintas, é necessário que as saídas sejam combinadas de forma a gerar um resultado único de percepção de conforto. Para tanto, um meta-classificador combina as árvores de decisão em um mesmo modelo. A Figura 3 mostra uma representação visual do modelo completo de classificação. As entradas para os modelos x, y e z são todos os dados de telemetria $\left(a_{1}, a_{2}, \ldots, a_{n}\right)$ e os dados de percepção de cada eixo $(x, y, z)$, respectivamente. O resultado são as saídas $x^{\prime}, y^{\prime}, z^{\prime}$, que são combinadas com os dados de percepção geral $g$ do passageiro. O resultado do meta-classificador é a classificação geral de conforto da viagem, que considera as diferentes percepções dos diferentes passageiros. A técnica de meta-classificação utilizada neste trabalho é o stacking, onde é realizado o empilhamento de diferentes modelos em um modelo final que busca melhorar o resultado do modelo em si ou unir classificações de diferentes aspectos de um conjunto de dados em um único classificador.

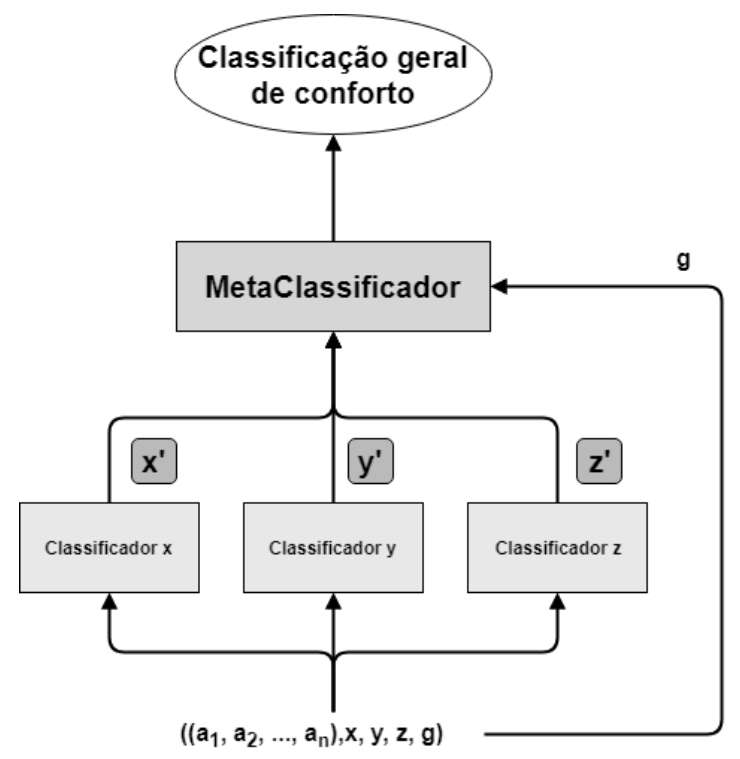

Figura 3: Modelo completo de classificação.

\section{APLICAÇÃO E ANÁLISE DOS RESULTADOS}

O método discutido nesse trabalho foi aplicado na cidade de Joinville, permitindo a coleta de dados reais para treinamento e avaliação. Os dados utilizados, tanto de telemetria, coletados por Bayer [16], quanto de percepção, coletados no âmbito do trabalho em tela, foram obtidos em parceria com uma das empresas responsáveis pelo transporte coletivo na cidade. O dispositivo de coleta foi instalado com autorização e apoio da empresa, assim como as percepções foram coletadas por colaboradores da própria empresa seguindo o protocolo definido nesta pesquisa.

A avaliação dos resultados dos treinamentos foi realizada por meio de uma análise quantitativa sobre o conjunto de teste previamente separado. Essa avaliação tem como objetivo a análise das acurácias dos modelos resultantes, buscando validar a abordagem proposta neste trabalho.

Considerando-se que o resultado dos treinamentos são 3 árvores de decisão, cada uma com 5 possíveis classes, foram selecionados alguns cenários específicos para a demonstração da generalização dos resultados. A avaliação é realizada sobre trajetórias de veículos com características semelhantes, mas anotações de percepção distintas. Para essa avaliação foram selecionadas trajetórias cujos percursos e horários fossem similares, de forma que fosse possível comparar os resultados em condições de trânsito minimamente equânimes. Cada cenário de teste é composto por duas coletas de percepção com anotações diferentes. Para cada árvore gerada pelo modelo um cenário é descrito. A avaliação busca analisar a acurácia da previsão dos modelos com relação a observações de passageiros que não foram utilizadas na indução dos modelos. 
Cada etapa descrita na seção 4 tem uma subseção dedicada à análise dos resultados, conforme apresentado a seguir.

\subsection{Dados coletados}

Os questionários de coleta de percepção foram aplicados na cidade de Joinville entre os dias 16 e 25 de setembro de 2017, em diferentes períodos do dia entre as 06 horas da manhã e as 11 horas da noite. Somente passageiros que viajaram no veículo equipado com o dispositivo de telemetria foram questionados.

Um total de 247 percepções de conforto de passageiros foram obtidas. Considerando que em Joinville cerca de 120 mil pessoas utilizam o transporte coletivo, é possível ter um nível de confiança de $95 \%$ e uma margem de erro de 6 pontos. A duração das viagens cujas percepções foram coletadas eram de cerca de 20 minutos, em média. Já o conjunto de dados de telemetria correspondente a esse período de coleta tem cerca 15 milhões de registros.

A Figura 4 apresenta as distribuições das percepções colhidas, onde o eixo horizontal apresenta as percepções estratificadas de conforto, de 1 a 5 , e o eixo vertical quantifica os respondentes com aquela percepção. A Figura 4.a, que tem o título Histograma de Frenagem, refere-se ao eixo de aceleração x. Já a Figura 4.b, que tem o título Histograma de Curvas, refere-se ao eixo de aceleração y. Por fim, a Figura 4.c, que tem o título Histograma de Trepidação, refere-se ao eixo de aceleração z. O conforto geral, representado pela Figura 4.d, que tem o título Histograma de Conforto Geral, representa as percepções do conforto da viagem como um todo.

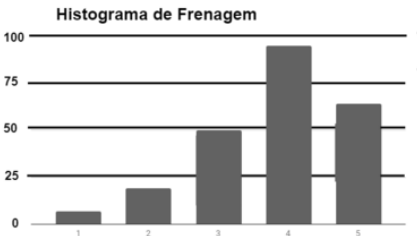

a)

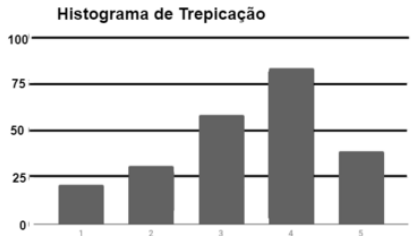

c)

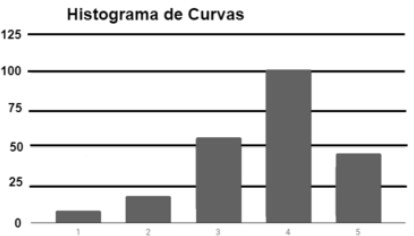

b)

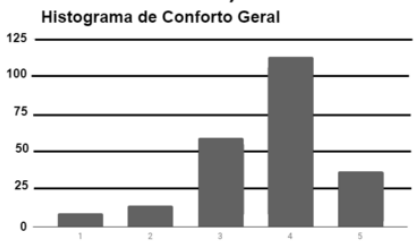

d)
Figura 4: Distribuição das percepções colhidas na aplicação do método.

\subsection{Preprocessamento}

Dada a característica de coleta assíncrona dos dados de telemetria, a coleta resultou em um arquivo esparso de dados. Entretanto, esse conjunto esparso precisou ser transformado em um conjunto denso de dados. Esse processamento foi feito com base no horário da coleta dos dados e sua discretização para instantes de um minuto. Em geral, os sensores têm diferentes frequências de coleta. Por exemplo, o sensor de GPS coleta a posição do veículo a cada minuto, enquanto que o acelerômetro realiza a coleta a cada 10 segundos. Dessa forma, para que o conjunto denso seja criado, para alguns sensores são realizadas interpolações de dados, enquanto que para outros são calculadas média e desvio padrão. Para os dados de acelerômetro utilizado no trabalho em tela foi utilizada a médias das acelerações coletadas a cada minuto. Após esse tratamento, o conjunto de dados denso tem cerca de 1 milhão e meio de instâncias.

Além da transformação do conjunto, é necessário que sejam retirados os dados que têm baixa relevância para o estudo. Portanto, o Algoritmo 1 tem como objetivo retirar os pontos cuja distância entre si é menor que um limite $d_{\max }$ pré-definido durante um curto período de tempo $t_{\max }$, considerando que a diferença entre as acelerações nesse ponto não sejam significativas, conforme um limite $a_{\text {max }}$ pré-definido. Os parâmetros utilizados para a aplicação desse algoritmo foram: $d_{\max }=5$ metros, $t_{\max }=4$ segundos, $a_{x} \max =a_{y} \max =a_{z} \max =0.1 \mathrm{~g}$. A aplicação desse algoritmo resultou em um conjunto de dados de cerca de 700 mil instâncias.

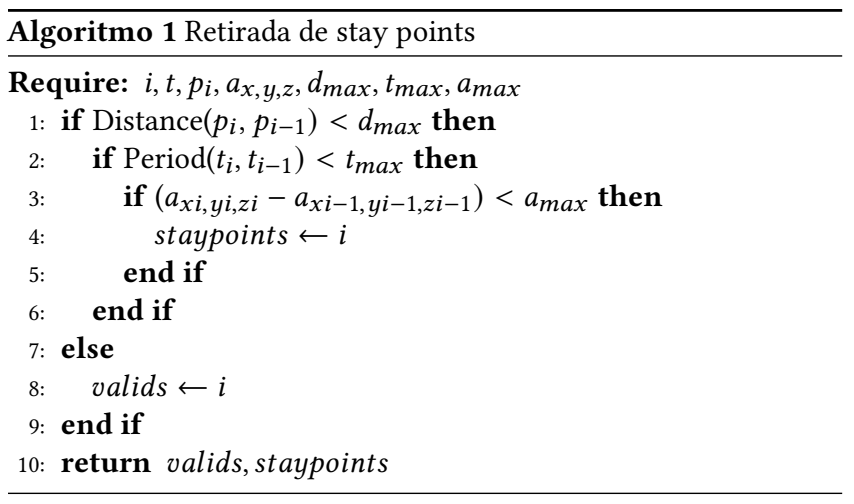

\subsection{Anotação dos dados de telemetria}

Após a retirada dos pontos inválidos, é necessário anotar os dados de telemetria com os dados de percepção dos usuários. Como mais de um passageiro fornece a percepção para um mesmo trajeto, é necessário, em primeiro lugar, realizar o consenso das percepções, de forma que cada trajeto tenha apenas uma percepção válida. Uma vez que esse processo é finalizado, é necessário realizar a anotação da telemetria. Para tanto, utiliza-se o processo descrito na seção 4.3.

O Algoritmo 2 descreve a forma de realização do consenso. Ele recebe como entrada um conjunto de percepções $P E$ e identifica em quais períodos existe sobreposição de coleta de percepção, então, calcula o consenso nesse período. Cada percepção é uma tupla $p e_{i}=$ $\left(h e_{i}, h d_{i}, p t_{i}, p c_{i}, p a_{i}, p g_{i}\right.$ ), composta pelo horário de embarque $h e_{i}$, horário de desembarque $h d_{i}$, percepção de conforto de trepidações $p t_{i}$, percepção de conforto de curvas $p c_{i}$, percepção de conforto de acelerações e frenagens $p a_{i}$, e, finalmente, percepção de conforto geral $p g_{i}$.

Após a realização do consenso, das 247 percepções coletadas foram obtidas 72 instâncias de percepções em consenso. Dando sequência ao processo, essas percepções precisam ser anotadas no conjunto de dados de telemetria. Para tanto, utiliza-se o Algoritmo 3 , que recebe como entrada as percepções $P$ após o consenso e os dados de telemetria $T$.

Os dados de percepção são percorridos e, para cada tupla de percepção, os dados de telemetria ordenados pelo momento da coleta são avaliados, linhas 1 e 2 . Se o dado de telemetria ocorreu 


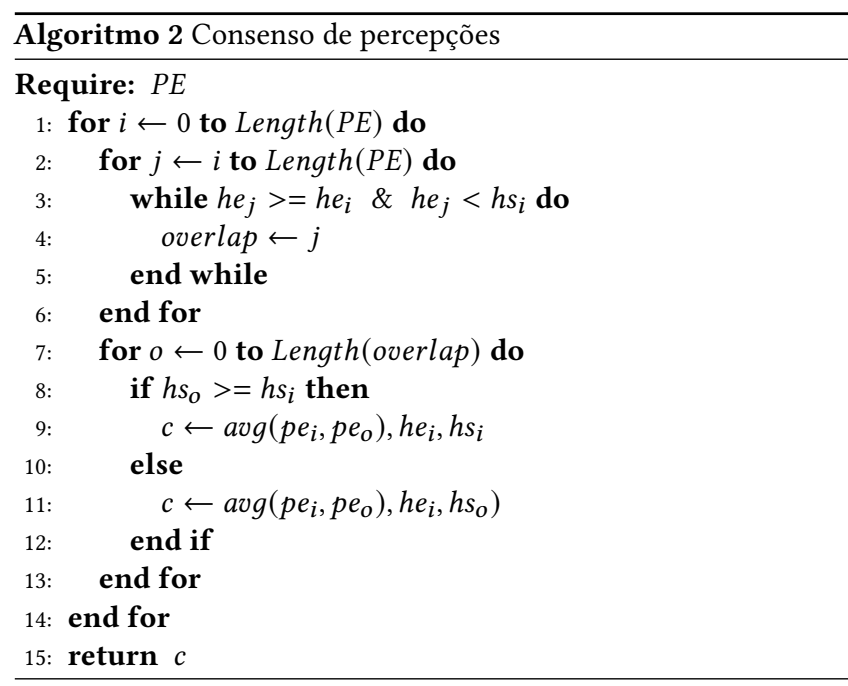

no período da tupla de percepção, então ele é armazenado até que algum dado não esteja mais nesse período, linhas 3 a 5 . A média e desvio padrão do conjunto é calculado, linhas 6 e 7, e todos os dados que estejam na área sob a curva de distribuição referente à percepção avaliada são anotados, linhas 9 a 38, conforme a Figura 2. O resultado do algoritmo de anotação é um conjunto de cerca de 15 mil instâncias.

\subsection{Treinamento das árvores de decisão}

Uma vez que os dados estão anotados, é possível realizar o treinamento e avaliação das árvores de decisão. $\mathrm{O}$ algoritmo escolhido foi o C4.5 [17], que utiliza a métrica de ganho de informação para o tratamento da sensibilidade de dados faltantes e que dá pesos diferentes para atributos com diferente importância no conjunto de dados. A implementação utilizada nesse trabalho é a J48, algoritmo contido na ferramenta Weka versão 3.8 [18].

Cada árvore de decisão foi treinada com o mesmo conjunto de dados, porém, cada uma utilizou a classe alvo do treinamento, eixo $\mathrm{x}, \mathrm{y}$ e $\mathrm{z}$ de aceleração, respectivamente. Os dados para o treinamento foram selecionados de forma que cada classe de conforto correspondesse a cerca de $20 \%$ do conjunto, na tentativa de manter o conjunto equilibrado e evitar o overfit para alguma classe em particular. A classe com menor quantidade de instâncias é a de MuitoDesconfortável, para todos os eixos, uma vez que as respostas para essa classe foram muito menos frequentes que para as outras classes, conforme Figura 4. Essa classe representa cerca de $15 \%$ do conjunto de treinamento.

Como a proposta deste trabalho é a utilização de uma metaclassificador, esse será o foco da análise de resultados, ainda que sejam comentados os resultados de cada classificador separadamente.

5.4.1 Treinamento dos classificadores intermediários. As três árvores, relacionadas com cada eixo de aceleração tiveram o seguinte desempenho: a árvore de conforto vertical, relacionada com o eixo $\mathrm{x}$ de aceleração, teve $82,3 \%$ de acurácia; a árvore de conforto lateral, relacionada com o eixo y, teve $77,2 \%$ de acurácia e a árvore de conforto horizontal, relacionada com o eixo z, teve $79 \%$ de acurácia.

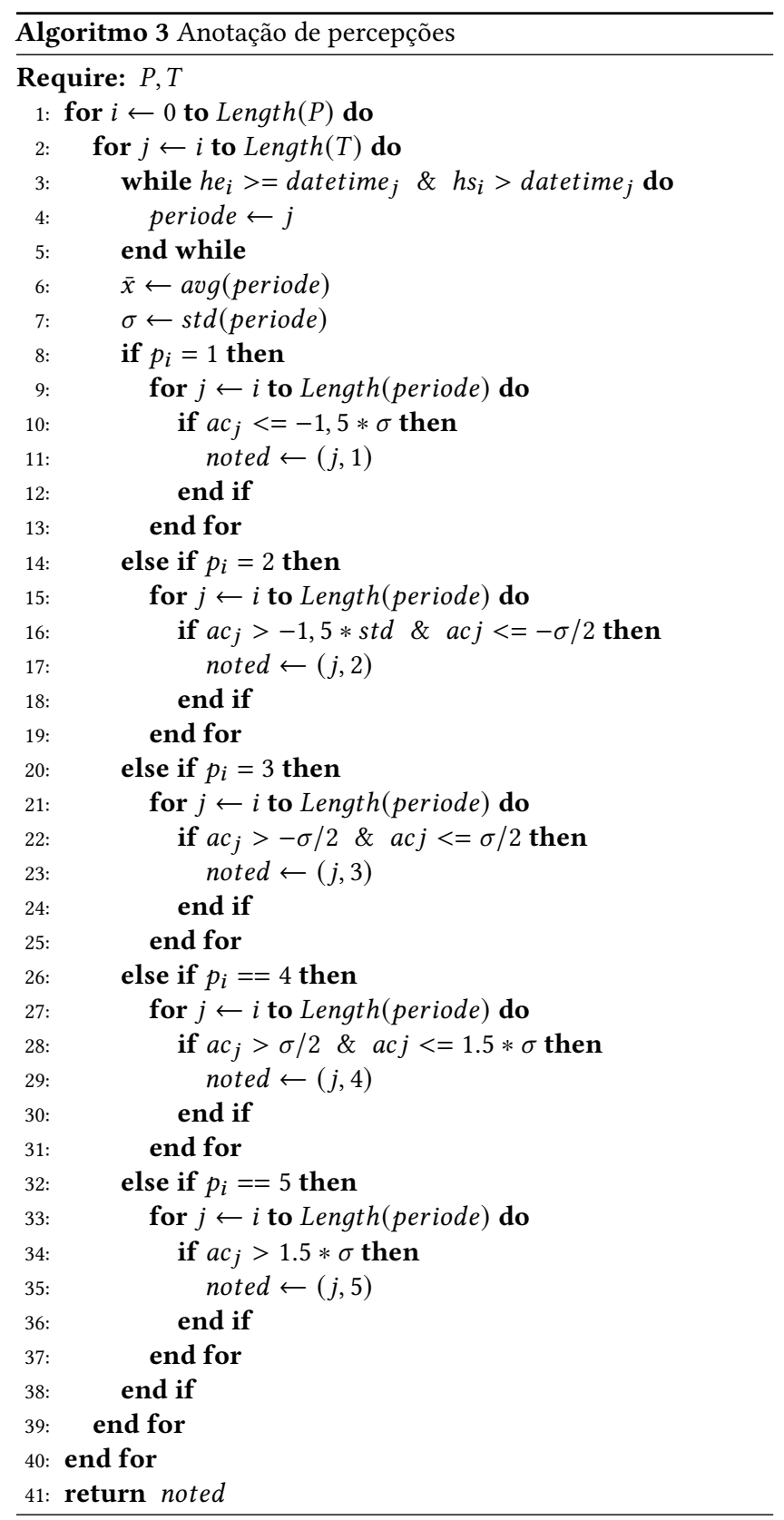

De um modo geral, a classe MuitoDesconfortavel, para cada um dos eixos, teve o pior desempenho nos testes. Esse resultado era esperado, uma vez que essa era a classe com menor quantidade de dados para o treinamento. Além disso, a acurácia acima de $70 \%$ das três árvores é uma evidência de que elas não sofreram overfitting.

5.4.2 Treinamento do meta classificador. Esse treinamento implica na entrada dos dados já classificados pelos outros modelos, bem como a classificação geral da viagem, também provinda pelos passageiros. Essa árvore teve acurácia de $86,3 \%$. Esse resultado é superior ao obtido por meio dos modelos intermediários, o que evidencia que sua utilização melhora a generalização dos resultados. 
A título de comparação, uma árvore foi treinada para a classificação do conforto geral dos passageiros contendo dos os dados de treinamento, portanto, sem o uso de um meta-classificador. Nesse teste, o modelo teve acurácia de 57\%, um resultado consideravelmente inferior ao alcançado pelo meta-classificador proposto. Dessa forma, é possível perceber que a utilização de modelos especializados em cada aspecto, agrupados em um meta-modelo, tem resultado superior ao de um modelo generalista que considera todos os dados de um único contexto.

\subsection{Avaliação dos modelos resultantes}

A avaliação ocorre por meio da previsão da classe de cada instância e a comparação com a classe esperada, além da observação da distribuição do erro na matriz de confusão correspondente ao modelo. Não é esperado que os cenários de avaliação apresentem acurácia idêntica ao treinamento, uma vez que os dados utilizados na avaliação são diferentes.

5.5.1 Cenário 1: Avaliação do modelo de conforto relacionado às trepidações. Para essa avaliação foram selecionadas duas trajetórias, no mesmo percurso, de cerca de $6,5 \mathrm{~km}$ percorridos, colhidas entre as 10 e 11 horas da manhã. Uma foi anotada como MuitoConfortavel e a outra como MuitoDesconfortavel. Essas instâncias têm um total de 1.482 pontos anotados com percepção utilizados para o teste.

A matriz de confusão relacionada ao teste é ilustrada pela Tabela 1. As colunas estão relacionadas com a quantidade de instâncias classificadas na classe representada, em que a letra $a$ representa a classe MuitoDesconfortavel, a letra $b$ representa a classe RazoavelmenteDesconfortavel, a letra $c$ representa a classe Confortavel, a letra $d$ a classe RazoavelmenteConfortavel, e a letra $e$ a classe MuitoConfortavel. Por sua vez, as linhas representam as classes esperadas para todas as instâncias contidas naquela linha. O melhor resultado possível é a observação de instâncias apenas nas classes cuja instância foi anotada, nesse caso $a$ e $e$, MuitoDesconfortavel e MuitoConfortavel, respectivamente.

TABELA 1: Matriz de confusão do teste da árvore de $x$ no Cenário 1

\begin{tabular}{lllcc|l}
\hline $\mathrm{a}$ & $\mathrm{b}$ & $\mathrm{c}$ & $\mathrm{d}$ & $\mathrm{e}$ & Classificado/ Esperado \\
\hline 598 & 33 & 26 & 27 & 7 & $\mathrm{a}=$ MuitoDesconfortavel \\
\hline 0 & 14 & 53 & $\begin{array}{c}68 \\
\text { Fonte: Tabela gerada pela autora. }\end{array}$
\end{tabular}

Nesse cenário, o modelo teve acurácia de $84,6 \%$, o que é ligeiramente superior à acurácia do treinamento da árvore, que foi de 82,3\%. É possível perceber que o erro nesse cenário é bem distribuído entre as classes centrais, e baixo nas classes de extremos. Além disso, existe um erro maior na classe MuitoDesconfortavel, o que é esperado, uma vez que essa classe tem menos instâncias de treinamento. Essa árvore tem uma generalização adequada, apesar do fato de que a maior parte do erro está em uma classe adjacente à classe objetivo, ou seja, na classe MuitoConfortavel e metade do erro está na classe RazoavelmenteConfortavel. Isso demonstra que existem diversas instâncias em que o modelo não tem robustez suficiente para distinguir completamente entre duas classes similares.
5.5.2 Cenário 2: Avaliação do modelo de conforto relacionado às curvas. Para essa avaliação foram selecionadas duas trajetórias, no mesmo percurso, de cerca de 3,5 km, colhidas entre as 13 e 14 horas da tarde. Uma anotada como MuitoConfortavel e a outra como MuitoDesconfortavel. Essas instâncias têm um total de 1.327 pontos anotados com percepção. A matriz de confusão relacionada ao teste é ilustrada pela Tabela 2. Essa tabela é organizada da mesma maneira que a descrita no primeiro cenário avaliado.

TABELA 2: Matriz de confusão do teste da árvore de $y$ no Cenário 2

\begin{tabular}{lllcc|l}
\hline $\mathrm{a}$ & $\mathrm{b}$ & $\mathrm{c}$ & $\mathrm{d}$ & $\mathrm{e}$ & Classificado/ Esperado \\
\hline 459 & 28 & 72 & 34 & 0 & $\mathrm{a}=$ MuitoDesconfortavel \\
\hline 6 & 12 & 65 & $\begin{array}{c}97 \\
\text { Fonte: Tabela gerada pela autora. }\end{array}$
\end{tabular}

Nesse cenário, a acurácia foi ligeiramente inferior à observada no treinamento, $76,3 \%$ do treinamento contra $77,2 \%$ do teste. Observase que aqui o erro é menos distribuído entre as classes. Para as instâncias classificadas como MuitoDesconfortavel, mais da metade do erro está concentrado na classe central. É possível que esse resultado esteja relacionado com a menor quantidade de instâncias de teste para a classe MuitoDesconfortavel, o que dificulta a classificação para essa classe. Ainda assim, essa instância tem acurácia de $77,4 \%$, superior à acurácia do treinamento do modelo.

Além disso, a classe MuitoConfortavel apresenta acurácia de $75,5 \%$, quase dois pontos percentuais abaixo da acurácia do modelo. Ainda, é possível perceber que o erro dessa classe está concentrado na classe RazoavelmenteConfortavel, que é uma classe adjacente à classe objetivo, demonstrando, novamente, que existe uma dificuldade de discernimento entre classes similares.

5.5.3 Cenário 3: Avaliação do modelo de conforto relacionado às acelerações e frenagens. Para essa avaliação foram selecionadas duas trajetórias, no mesmo percurso, de cerca de $5 \mathrm{~km}$, colhidas entre as 13 e 14 horas da tarde. Uma anotada como MuitoConfortavel e a outra como MuitoDesconfortavel. Essas instâncias têm um total de 1.567 de pontos anotados com percepção. A matriz de confusão desse cenário está representada na Tabela 3 e é organizada da mesma maneira que a descrita nos cenários anteriores.

TABELA 3: Matriz de confusão do teste da árvore de $z$ no Cenário 3

\begin{tabular}{lllcc|l}
\hline $\mathrm{a}$ & $\mathrm{b}$ & $\mathrm{c}$ & $\mathrm{d}$ & $\mathrm{e}$ & Classificado/ Esperado \\
\hline 545 & 45 & 9 & 51 & 29 & $\mathrm{a}=$ MuitoDesconfortavel \\
\hline 16 & 26 & 42 & 86 & 718 & $\mathrm{e}=$ MuitoConfortavel \\
& & \multicolumn{4}{c}{ Fonte: Tabela gerada pela autora. }
\end{tabular}

Esse cenário teve acurácia de 80,7\%, superior à acurácia de $79 \%$ observada no treinamento. Ainda que o resultado seja melhor que o do treinamento, ele tem o maior erro na classe extrema da classe objetivo, ou seja, para a classe MuitoDesconfortavel, esse cenário apresenta mais de $20 \%$ do erro na classe MuitoConfortavel, e quase $10 \%$ na situação contrária. Além disso, o erro se distribui por todas as classes de forma quase uniforme. Isso demonstra que o modelo 
pode ter dificuldade de generalização para outras instâncias que tenham uma quantidade menor de observações, uma vez que a distribuição e anotação dessas observações pode deixar os limites entre as classes menos perceptíveis.

Por fim, a classe MuitoConfortavel apresenta novamente a maior parte do erro em uma classe adjacente. Ainda assim, essa classe tem acurácia similar à acurácia do treinamento, $80,9 \%$ contra $80,7 \%$.

5.5.4 Cenário 4: Avaliação do meta-modelo de conforto. Para essa avaliação foram selecionadas duas trajetórias, no mesmo percurso, de cerca de $6,5 \mathrm{~km}$, colhidas entre as 13 e 14 horas e 30 minutos da tarde. Uma anotada como MuitoConfortavel e a outra como MuitoDesconfortavel. Essas instâncias têm um total de 3.327 pontos anotados com percepção. A matriz de confusão é representada pela Tabela 4, que deve ser interpretada como nos cenários anteriores.

TABELA 4: Matriz de confusão do teste da árvore de $x$ no Cenário 4

\begin{tabular}{lllll|l}
\hline $\mathrm{a}$ & $\mathrm{b}$ & $\mathrm{c}$ & $\mathrm{d}$ & $\mathrm{e}$ & Classificado/ Esperado \\
\hline 1259 & 66 & 74 & 31 & 43 & $\mathrm{a}=$ MuitoDesconfortavel \\
0 & 75 & 48 & 54 & 1577 & $\mathrm{e}=$ MuitoConfortavel \\
\hline \multicolumn{5}{c}{ Fonte: Tabela gerada pela autora. }
\end{tabular}

Esse cenário é aplicado sobre o meta-modelo de classificação, que teve uma acurácia de $86,3 \%$ no treino. O cenário tem acurácia de $85,2 \%$, ligeiramente inferior ao treino, mas superior às acurácias de cada um dos modelos intermediários. Observa-se que o metamodelo tem erro bem distribuído entre as classes. Além disso, 20\% do erro da classe MuitoDesconfortavel está concentrado na classe MuitoConfortavel, que é a classe diametralmente contrária à classe esperada.

O resultado desse modelo demonstra que a utilização de um empilhamento de modelos especialistas, por meio de um meta-modelo generalista tem efeito positivo nos resultados das classificações. Além disso, os modelos intermediário consideram como o conjunto de dados coletado influencia na classe esperada, enquanto que o meta-modelo pode se utilizar dessas classes já previamente classificadas para obter resultados mais completos e com melhor generalização para os conjuntos de testes.

\section{CONCLUSÃO E TRABALHOS FUTUROS}

Esse trabalho propôs a construção de um modelo capaz de generalizar a percepção de conforto de passageiros do transporte coletivo, usando como base dados de telemetria do veículo e a percepção de conforto do próprio passageiro. Para alcançar tal objetivo foram utilizadas técnicas de aprendizado de máquina que pudessem induzir modelos de classificação do tipo árvores de decisão.

A fim de classificar os dados, foram treinadas 4 árvores de decisão, 3 chamadas de modelos intermediários, relacionadas com os eixos de aceleração, e 1 chamado de meta-modelo, que se utiliza da classificação gerada pelas árvores intermediárias para a classificação do conforto geral. Tanto as árvores intermediárias quando o meta-classificador têm desempenho adequado, mas a utilização do meta-classificador melhorou significativamente o resultado. A maior parte das instâncias de teste foram classificadas corretamente, com baixa taxa de confusão entre as classes adjacentes.
Uma das limitações desse modelo está relacionada a forma com a qual os dados foram anotados. Como os dados foram anotados com base na distribuição das coletas, não é possível afirmar que a percepção de conforto atribuída pelo passageiro esteja em consonância com os dados anotados. Além disso, o modelo não considera que o conforto pode ser afetado por aspectos dependentes de ordem de ocorrência. Portanto, é necessário melhorar e expandir o modelo para a utilização de algum classificador que considere a ordem e as influências entre as diferentes características de uma viagem ao longo do tempo.

Os trabalhos futuros incluem a aplicação do meta-modelo considerando mais classificadores intermediários, na tentativa de apurar ainda mais o resultado obtido. Além disso, a utilização de outras técnicas de classificação dos dados, como modelos estatísticos que consideram as influências dos dados entre si em um mesmo momento e em momentos passados, permitindo que tais influências sejam destacadas e tenham peso no resultado final da classificação.

\section{AGRADECIMENTOS}

Este trabalho foi parcialmente financiado pela Fundação de Amparo à Pesquisa e Inovação do Estado de Santa Catarina (FAPESC).

\section{REFERENCES}

[1] CNT. Anuário cnt do transporte - estatísticas consolidadas 2017, 2017. URL https://anuariodotransporte.cnt.org.br/2017/File/PrincipaisDados.pdf.

[2] Nick Cohn. Tomtom traffic index: Toward a global measure. ITS France 2014, 2014.

[3] Tariq Van Rooijen and Hans Quak. City logistics in the european civitas initiative. Procedia-Social and Behavioral Sciences, 125:312-325, 2014.

[4] Markus Fellesson and Margareta Friman. Perceived satisfaction with public transport service in nine european cities. In Fournal of the Transportation Research Forum, volume 47, 2012.

[5] Mohammad Nurul Hassan, Yaser E Hawas, and Kamran Ahmed. A multidimensional framework for evaluating the transit service performance. Transportation Research Part A: Policy and Practice, 50:47-61, 2013.

[6] Satu Innamaa and Merja Penttinen. Impacts of a green-driving application in city buses on fuel consumption, speeding and passenger comfort. IET Intelligent Transport Systems, 8(5):435-444, 2013.

[7] Juan C Castellanos and Fabiano Fruett. Embedded system to evaluate the passenger comfort in public transportation based on dynamical vehicle behavior with user's feedback. Measurement, 47:442-451, 2014.

[8] Kai Zhang, Kan Zhou, and Fangzhou Zhang. Evaluating bus transit performance of chinese cities: developing an overall bus comfort model. Transportation Research Part A: Policy and Practice, 69:105-112, 2014.

[9] Laura Eboli, Gabriella Mazzulla, and Giuseppe Pungillo. Measuring bus comfort levels by using acceleration instantaneous values. Transp. Res. Procedia, 18(June): 27-34, 2016.

[10] Solange Oliveira Rezende. Sistemas inteligentes: fundamentos e aplicações. Editora Manole Ltda, 2003.

[11] Yu Zheng. Trajectory data mining: an overview. ACM Transactions on Intelligent Systems and Technology (TIST), 6(3):1-41, 2015.

[12] Fosca Giannotti, Mirco Nanni, Fabio Pinelli, and Dino Pedreschi. Trajectory pattern mining. In Proceedings of the 13th ACM SIGKDD international conference on Knowledge discovery and data mining, pages 330-339, 2007.

[13] V Bogorny and FJ Braz. Introdução a trajetórias de objetos móveis: conceitos, armazenamento e análise de dados. Univille, 2012.

[14] João Barcelos Neto. Mecânica Newtoniana, Lgrangiana e Hamiltoniana. Editora Livraria da Física, 2004.

[15] Jinhan Park, Junwoo Lee, Sejin Ahn, and Weuibong Jeong. Reduced ride comfort caused by beating idle vibrations in passenger vehicles. International fournal of Industrial Ergonomics, 57:74-79, 2017.

[16] Flavio Roberto Bayer. Uma abordagem para identificação de perfis de motoristas do transporte coletivo com foco na minimização do consumo de combustível. 2017.

[17] J Ross Quinlan. C4. 5: programs for machine learning. Elsevier, 2014

[18] Eibe Frank, Mark A. Hall, and Ian H. Witten. The WEKA Workbench. Online Appendix for "Data Mining: Practical Machine Learning Tools and Techniques". Morgan Kaufmann, 4th edition, 2016. 